\title{
Loss mechanism of all-fiber cascaded side pumping combiner
}

\author{
Chengmin Lei ${ }^{1}$, Zilun Chen ${ }^{1,2,3}$, Yanran $\mathrm{Gu}^{1}, \mathrm{Hu}_{\mathrm{Xiao}}{ }^{1,2,3}$, and Jing Hou ${ }^{1,2,3}$ \\ ${ }^{1}$ College of Advanced Interdisciplinary Studies, National University of Defense Technology, Changsha 410073, China \\ ${ }^{2}$ Hunan Provincial Key Laboratory of High Energy Laser Technology, National University of Defense Technology, \\ Changsha 410073, China \\ ${ }^{3}$ Hunan Provincial Collaborative Innovation Center of High Power Fiber Laser, National University of Defense Technology, Changsha \\ 410073, China \\ (Received 10 July 2018; revised 31 August 2018; accepted 26 September 2018)
}

\begin{abstract}
Compared with end pumping fiber combiner, one of the advantages for side pumping combiner is the unlimited pumping points, which means multi-point or cascaded side pumping can be realized. However, the loss mechanism of the cascaded structure is rarely discussed. In this paper, we present the numerical and experimental investigation about the loss mechanism of a two-stage-cascaded side pumping combiner based on tapered-fused technique. The influence of loss mechanism on the coupling efficiency and thermal load of the fiber coating is analyzed according to simulations and experiments with different tapering ratios for the first stage. Based on the analysis, a cascaded component with total pump coupling efficiency of $96.4 \%$ handling a pump power of $1088 \mathrm{~W}$ is achieved by employing $1018 \mathrm{~nm}$ fiber laser as the pump source. Future work to further improve the performance of a cascaded side pumping combiner is discussed and prospected.
\end{abstract}

Keywords: fiber combiner; fiber laser; side pumping

\section{Introduction}

Fiber lasers have seen progressive development and been widely applied in industrial field, defense technology and medical science over the past several decades. The investigation of all-fiber components for the highly integrated high power fiber laser and amplifier systems has been increased in recent years. One of the most key components is a pump/signal combiner. The side pumping combiner, in which the pump light is coupled into the double-clad fiber through the fiber side, is of great interest in these two decades. The attractive characteristics of side pumping combiner, such as uninterrupted signal fiber core and unlimited pump points, which an end pumping combiner (such as tapered-fused bundle (TFB) technique ${ }^{[1]}$ ) cannot achieve, facilitate their potential applications in fiber laser and amplifiers. Among the reported side pumping couplers, those in $3 \mathrm{D}$ structure such as adhered micro-prism ${ }^{[2]}, \mathrm{V}_{\text {-groove }}{ }^{[2,3]}$, embedded mirror ${ }^{[4]}$ and diffraction grating ${ }^{[5,6]}$, restricted the further improvement of the stability and compactness of

Correspondence to: Z. Chen, College of Advanced Interdisciplinary Studies, National University of Defense Technology, Changsha 410073, China. Email: zilun2003@163.com the system, as well as the pump power handling capability (only W level to $10 \mathrm{~W}$ level) and pump coupling efficiency (not higher than 90\%). The all-fiber structure has seen more advantages. A tapered-fused method, which is based on direct fusion of one or several tapered pump fibers to the surface of a double-clad signal fiber, seems to be a more potential method because of its high coupling efficiency and the capability of handling high pump power up to $\mathrm{kW}$ class $^{[7-11]}$.

In order to further increase the output power from the fiber laser, higher pumping power coupled into double-clad signal fiber is required. Since the pumping points in a side pumping scheme are not limited, multi-point or cascaded side pumping can be realized. Tan et al. reported the cascaded combiner ${ }^{[12]}$, consisting of five combiners by using tapered-fused technique, and constructed a bi-directionally pumped fiber laser oscillator with $780 \mathrm{~W}$ output power when injecting $1096 \mathrm{~W}$ pump power. The laser was pumped via intra-cavity fiber-cascaded combiners and extra-cavity fibercascaded combiners. This work showed the advantage of side pumping technique that it enhances the pump power through cascaded structure as well as the potential of multipoint distributed pumping. However, compared with TFB 
end pumping combiner which can easily achieve $(N+$ $1) \times 1$ structure $(N$ can be 7 or more) with high coupling efficiency for every pump fiber ${ }^{[13-15]}$, for a tapered-fused side pumping combiner, the increase of pump fibers and high pump coupling efficiency requirement of the components cannot be achieved simultaneously as illustrated in some experimental work. The increased number of pump fibers at one pump point was proven to result in a decrease of pump coupling efficiency in Ref. [7]. This is why a $(2+$ 1) $\times 1$ structure is more advisable for high pump coupling efficiency requirement, while using cascaded scheme by inserting more pump fibers at several positions along the signal fiber will also reduce the coupling efficiency and insert more signal loss and beam quality degradation ${ }^{[12]}$. Since in a cascaded scheme, different side combiners will influence each other on pump coupling efficiency and pump power loss, it is of great importance to figure out the pump power loss mechanism in a cascaded scheme and find out a balance between the increase of pump arms and high coupling efficiency. Though this effect has been pointed out phenomenally, the causes and physical mechanism of extra pump power loss for side pumping combiners in cascaded structure were rarely investigated and analyzed thoroughly.

In this paper, we report simulations and experiments about the pump power loss mechanism of two-cascaded side pumping combiner based on tapered-fused technique for the first time. First, the optical design and numerical modeling of two-cascaded combiner are depicted. Then, simulation results for different parameters of the two combiners are shown in Section 2. These simulation results can be used as an instruction to the parameter selection and optimization during the fabrication of the components. Then, the experiment of the two-cascaded $(1+1) \times 1$ combiners with different parameters is demonstrated. Last, a cascaded component is used for handling a pump power of $1088 \mathrm{~W}$ when employing $1018 \mathrm{~nm}$ fiber laser as the pump source, with total pump coupling efficiency of $96.4 \%$.

\section{Theoretical analysis}

\subsection{Numerical model}

The numerical simulation is carried out by beam propagation method (BPM), which is a method for solving the Helmholtz equation for optical fibers ${ }^{[16]}$. The schematic diagram of the numerical model for the two-cascaded combiner is shown in Figure 1, which is composed by two $(1+1) \times 1$ side pumping combiners. The core/cladding diameter of the double-clad signal fiber is 20 and $400 \mu \mathrm{m}$, respectively, with a cladding numerical aperture (NA) of 0.46 . The core/cladding diameter of two pump fibers is $220 / 242 \mu \mathrm{m}$ with NA $=0.22$. $L_{\text {taper }}=9 \mathrm{~mm}$ and $L_{\text {waist }}=1 \mathrm{~mm}$ are the lengths of the transition region and the taper waist. The diameter of the taper waist is defined as $D_{\text {waist. }}$. As for the

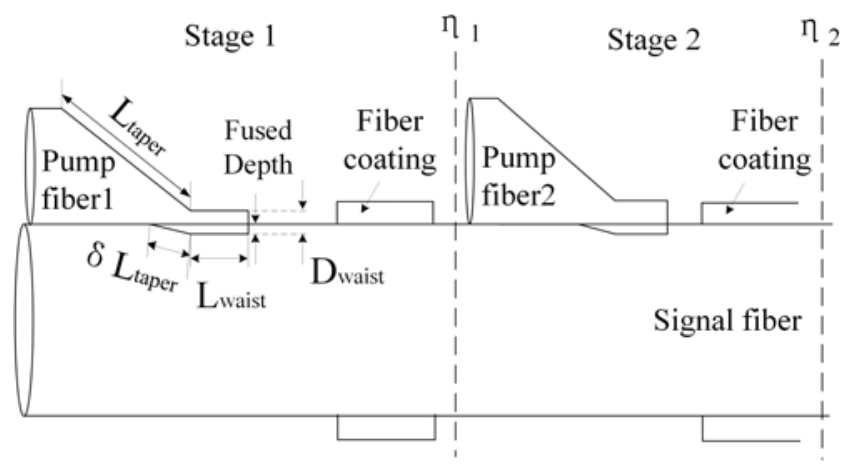

Figure 1. The longitudinal scheme of the cascaded combiner.

geometrical shape of the taper in the longitudinal direction, for simplicity, a linear shape is assumed in the simulations instead of the parabolic shape. Since the simulation of side pumping combiner with the consideration of fused depth may be more reliable as discussed in our previous work ${ }^{[10]}$, similarly, in this numerical model, we assume that the fused depth increases gradually along a length of $\delta L_{\text {taper }}$ and reaches the maximum at the taper waist (this maximum depth is defined as fused depth in the calculation). The distance between the coating edge and the taper waist end of the pump fiber is assumed to be $5 \mathrm{~mm}$ and the coating length for every combiner to be $5 \mathrm{~mm}$. Fused depth for both stages is $5 \mu \mathrm{m}$. Detailed information about the parameter setting and numerical model description of noncascaded side pumping combiner can be found in Ref. [10]. By using BPM, the integral power in different waveguides can be monitored along the longitudinal direction. The total power is set to 1. The pump wavelength is set to $976 \mathrm{~nm}$.

The analysis of the loss mechanism for a noncascaded side pumping combiner by tapered-fused method has been proposed and well-discussed recently. The simulations about the influence of taper ratio, taper length, pump light input NA and number of pump ports on the pump efficiency and loss mechanism were performed by using the ray tracing method in Ref. [7]. Furthermore, we first calculated the evolution of the leakage power into the coating of the signal fiber (LPC) along the coating length, which is the most critical pump power loss of the side pumping combiner. However, in terms of a cascaded structure as shown in Figure 1, when the pump light (inserted at Pump fiber 1) that has already coupled into the inner cladding of the signal fiber through the coupling region of Stage 1 propagates to Stage 2, it may introduce extra power loss due to the irregular waveguide shape in its coupling region, giving rise to a reduction of total coupling efficiency. Hence, it is of high importance to explore how the pump light which is inserted from the pump arm at the former-stage combiner propagates in the cascaded scheme, especially in the latter stage. In our numerical simulation, we mainly calculate the coupling efficiency at Stage $1\left(\eta_{1}\right)$ and Stage $2\left(\eta_{2}\right)$, LPC for Stage 1 and for Stage 2 as well as 
the power loss along the coupling region of Stage 2, under the condition of launching pump light from Pump fiber 1 . More specifically, $\eta_{1}$ and $\eta_{2}$ refer to the integral power over the waveguide cross section at the longitudinal position of the end of coating at Stages 1 and 2. In the simulation work of Ref. [10], to further understand LPC, the power evolution of this loss along the coating length was calculated and analyzed for the first time. In this paper, in order to better understand the influence of structure parameters on LPC of every stage, we only present its average value along the coating length.

\subsection{Power loss analysis in Stage 2}

Figure 2(a) shows the influence of $D_{\text {waist }}$ of Stage 1 on the pump coupling efficiency $\eta_{1}$ and $\eta_{2}$ from Stage 1. Since the pump fiber is a multi-mode fiber, we randomly select a higher-mode $\mathrm{LP}_{33}$ as the launch field of the simulation. At a constant $D_{\text {waist }}$, a decrease of coupling efficiency can be seen after the propagation of Stage 2, as shown in Figure 2(a). For instance, for $D_{\text {waist }}=20 \mu \mathrm{m}, \eta_{1}$ of $96.84 \%$ is obtained while the coupling efficiency after Stage 2 is calculated to be $92.48 \%$. This can be explained by two types of power loss, one of which is power loss along the coupling region of Stage 2. As shown in Figure 2(b), for monitoring this type of loss, the evolution of the waveguide power in Pump fiber 2 and the inner cladding of signal fiber as a percentage to the total pump power is calculated when $D_{\text {waist }}$ is $10 \mu \mathrm{m}$ and $20 \mu \mathrm{m}$. The longitudinal length of $0 \mathrm{~mm}$ in Figure 2(b) refers to the beginning position of Pump fiber 2 as shown in Figure 1. It can be observed that there is a tiny portion of power leaking into Pump fiber 2. This can be explained by the fact that the propagation along the coupling region of Stage 2 will lead to an increase of propagation angle of the pump light, and as long as the propagation angle exceeds the internal total reflection angle, pump power might then leak into the ambient air. Otherwise, on the condition that internal total reflection is satisfied, these kinds of pump light rays are leaked into the air at the end facet of Pump fiber 2.

Another type of power loss arises from LPC at the signal fiber coating of Stage 2. Figure 2(c) presents the comparison of average monitored power at the coating layer of two stages as a percentage of the input pump power, depending on $D_{\text {waist }}$ of Stage 1. In general, it can be seen that LPC2 is relatively higher than LPC1, under the same $D_{\text {waist }}$ of Stage 1 (or the same structure parameter of the component). In Stage 1, for the pump light coupling into the signal fiber successfully, as we analyzed before ${ }^{[10]}$, if pump light NA exceeds the cladding NA of the signal fiber, this pump power will couple into the coating of the signal fiber and become LPC1. However, for the light in signal fiber that propagates to Stage 2, the coupling region of Stage 2 will lead to an increased NA of the pump light. Since LPC will evidently increase with the pump light $\mathrm{NA}^{[7]}, \mathrm{LPC} 2$
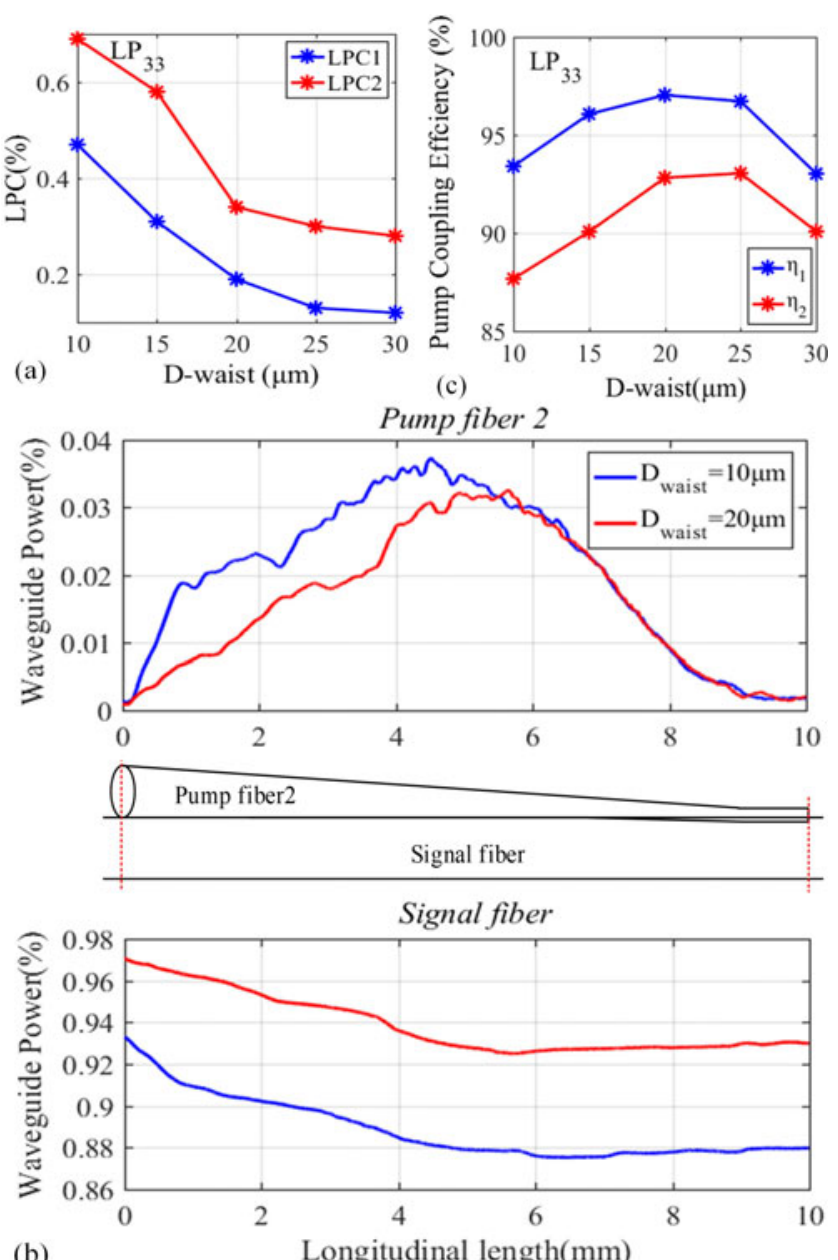

(b)

Longitudinal length(mm)

Figure 2. (a) $\eta_{1}, \eta_{2}$ with respect to $D_{\text {waist }}$ of Stage 1. (b) The evolution of waveguide power in Pump fiber 2 and signal fiber (inner cladding) to the total pump power along the length of Pump fiber 2 of Stage 2 for $D_{\text {waist }}=$ $10 \mu \mathrm{m}$ and $20 \mu \mathrm{m}$. (c) LPC1, LPC2 with respect to $D_{\text {waist }}$ of Stage $1\left(\mathrm{LP}_{33}\right.$ launching at Stage $1, \delta=0.1, D_{\text {waist }}$ of Stage 2 is $20 \mu \mathrm{m}$ ).

appears to be higher than LPC1. Note that these results are only under the condition that pump power is launched from Stage 1 but no power is launched from Stage 2. Imaging that pump light inserted from the pump fibers of both stages in the experimental application, the original LPC in Stage 2, in addition with the enhanced LPC derived from Stage 1, will significantly increase the risk of heat accumulation at coating layer due to the greater absorption coefficient for near-infrared light, compared with quartz material, and then damage the component especially at high power level. Hence, compared with power loss along the coupling region of Stage 2 that can be handled by air or water cooling devices, LPC in Stage 2 is more critical and the structure parameters should be carefully designed to suppress it as much as possible.

It is also found that $D_{\text {waist }}$ of Stage 1 will influence the mentioned two types of loss. For example, as shown in Figure 2(b) (lower), a $D_{\text {waist }}$ of $10 \mu \mathrm{m}$ leads to the power 
decrease in signal fiber of $5.3 \%$ from $93.4 \%$ to $88.1 \%$, whereas for $D_{\text {waist }}$ of $20 \mu \mathrm{m}$, the power decrease of $4.0 \%$ is calculated along the coupling region. As for LPC2, a $D_{\text {waist }}$ of $10 \mu \mathrm{m}$ leads to LPC 2 of $0.69 \%$ while for $D_{\text {waist }}$ of $30 \mu \mathrm{m}$, only $0.29 \%$ of this power is calculated. A lower $D_{\text {waist }}$ of Stage 1, namely a higher taper ratio (the diameter of the cladding of pump fiber $/ D_{\text {waist }}$ ), will cause further increase of the pump light NA, thus leading to the growth of two types of loss when the light propagates to Stage 2. Therefore, in a two-cascaded structure, a large $D_{\text {waist }}$ of Stage 1 is desirable, for the purpose of cutting down the loss of pump power (launched from Stage 1) along the coupling region of Stage 2, at the same time reducing LPC at Stage 2 to avoid component damage during high power operation. Even so, a large $D_{\text {waist }}$ is also inadvisable due to the relatively lower coupling efficiency even at the first stage. Therefore, $D_{\text {waist }}$ must be carefully selected to satisfy the requirement of a cascaded component for both high pump coupling efficiency and high stability under high power level.

Next, we figure out how the structure parameter of Stage 2 influences the loss mechanism in the two-cascaded side pumping combiner. As discussed before, the pump light inserted from Stage 1 will experience an increase of NA when propagating through the coupling region of Stage 2, thus introducing power loss along the coupling region as well as evident LPC2, which means the waveguide shape at the coupling region of Stage 2 can also influence the loss mechanism in Stage 2. In contrast with fused depth and $D_{\text {waist }}$ of Stage 2, fused ratio $\delta$ is the structure parameter that more significantly influences the coupling coefficient between Pump fiber 2 and the signal fiber. As illustrated in Section 2.1, we assume that the fused depth increases gradually along a length of $\delta L_{\text {taper }}$ and reaches the maximum at the taper waist, which means $\delta$ is the structure parameter that can describe not only the longitudinal position of fused depth but also the size and extent of the fused region between the pump fiber and signal fiber. Thus, we mainly discuss the impact of $\delta$ at Stage 2 on the loss mechanism, keeping the structure parameters of Stage 1 unchanged. Figure 3(a) illustrates $\eta_{2}$ and LPC2 at different $\delta$ of Stage 2 with $\mathrm{LP}_{33}$ launching at Stage 1 and $D_{\text {waist }}$ at both stages to be $20 \mu \mathrm{m}$. As shown in Figures 2(a) and 2(c), with $\mathrm{LP}_{33}$ launching at Stage 1 and $D_{\text {waist }}$ at Stage being $20 \mu \mathrm{m}, \eta_{1}$ and LPC1 are calculated to be $97.1 \%$ and $0.18 \%$. In Figure 3(a), it can be seen that $\eta_{2}$ is increased with the rise of $\delta$. For example, $\delta=0.1$ leads to a $\eta_{2}$ of $92.5 \%$ while for $\delta=0.3, \eta_{2}$ of $93.1 \%$ is calculated. One reason is the decreased power loss along the coupling region of Stage 2, from 4.5\% (97.1\%-92.6\%) at $\delta=0.1$ to $3.9 \%(97.1 \%-93.2 \%)$ at $\delta=0.3$, as shown in Figure 3(b) (lower). Besides, from Figure 3(b) (upper) it can be seen that $\delta$ mainly influences the light propagation in the coupling region with fused depth introduced - for example, longitudinal length $>6.3 \mathrm{~mm}$ for $\delta=0.3\left(L_{\text {taper }}-\delta L_{\text {taper }}\right)$, rather than the beginning of the coupling region. Another reason for the growth of $\eta_{2}$ with $\delta$ from 0.1 to 0.3 can be
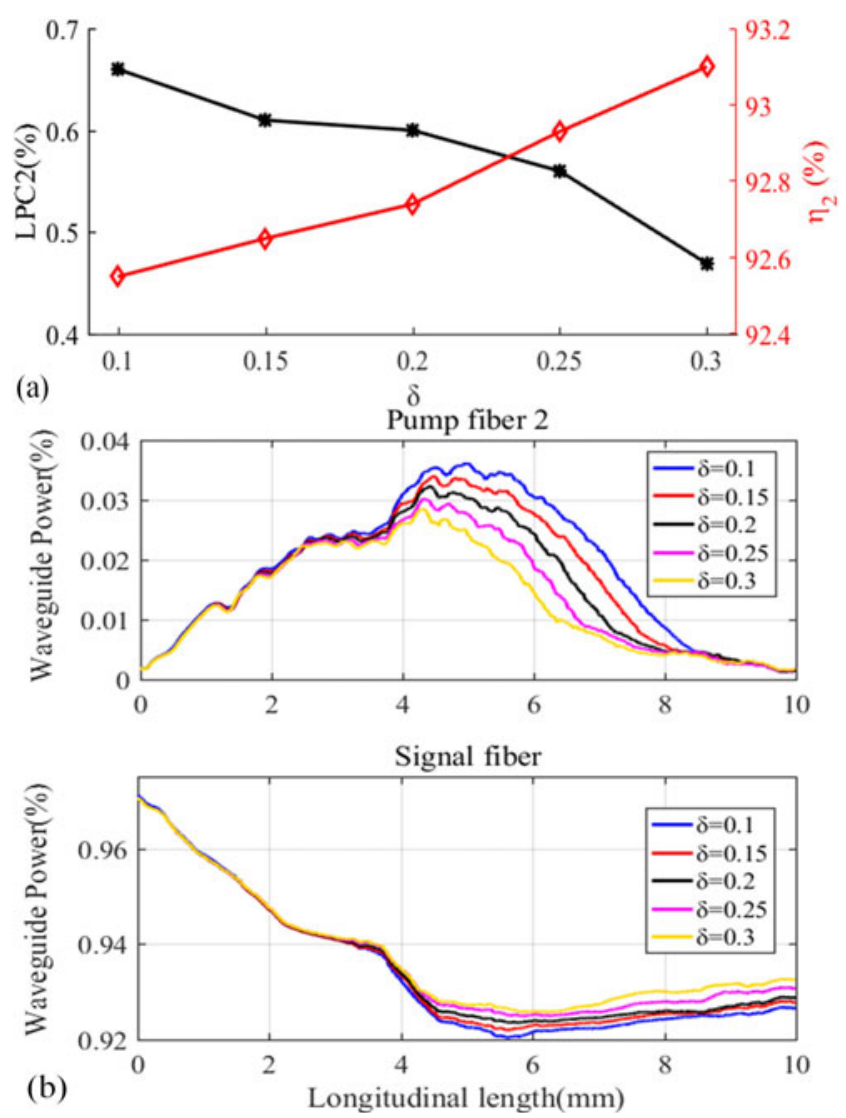

Figure 3. (a) $\eta 2$ and LPC with respect to $\delta$ of Stage 2. (b) The evolution of waveguide power in Pump fiber 2 and signal fiber (inner cladding) to the total pump power along the length of Pump fiber 2 of Stage 2 for different $\delta$ of Stage 2. (LP33 launching at Stage $1, D_{\text {waist }}$ (at both stages) is $20 \mu \mathrm{m}$.)

the decreased LPC2 to the total pump power from $0.67 \%$ to $0.47 \%$, as shown in Figure 3(a).

\subsection{Discussion and conclusion}

The simulation implies that first of all, the power loss coupling into the coating in Stage 2 is more serious than that in Stage 1, due to the enhanced LPC derived from light power launching in Stage 1, in conjunction with its own LPC when launching pump power in Stage 2. In the application of a cascaded side pumping combiner, it will take more effort and attention to handle the heat load on the signal fiber coating of the second stage rather than the first one, by improving the packaging design and cooling system of the component. Second, since $D_{\text {waist }}$ of the first stage strongly influences the loss mechanism of the component as analyzed, it must be carefully selected in the fabrication for the purpose of designing a component with high coupling efficiency as well as low thermal load on the fiber coating. Third, the increase of $\delta$ in Stage 2 is an effective approach to improve the coupling efficiency $\eta_{2}$ and suppress LPC2. During the fusion splicing of the component fabrication, $\delta$ can be 


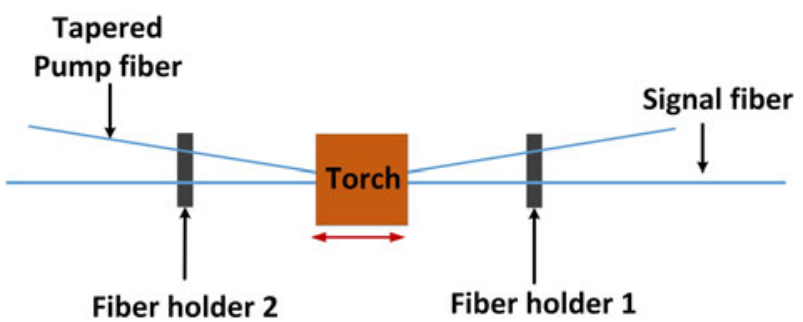

Figure 4. The schematic of the combiner fabrication system.

controllable by the size and scanning region of the heating torch, which means a torch with proper size is required and a scanning program of the torch is recommended to guarantee a sufficient fused region.

\section{Experimental results and discussion}

\subsection{Experimental setup}

The heat source for tapering and fusion splicing is a hydrogen-oxygen flame. The fabrication and online testing system, as well as the fabrication process of the combiner was described in our previous work in detail ${ }^{[10]}$. Figure 4 shows the schematic of the combiner fabrication system. The signal fiber and the tapered pump fiber are horizontally fixed by the fiber holders to avoid the deformation of the signal fiber. The signal fiber is fixed in the middle groove of the holder and the tapered pump fiber is directly spliced with the side of the signal fiber without stress and twist. Since the numerical results in Section 2.2 indicate that a scan program of the torch is advisable to guarantee an enough fused region, the heating torch is scanning with a scan speed of $0.5 \mathrm{~mm} / \mathrm{s}$ and scan length of $1 \mathrm{~mm}$ during the $50 \mathrm{~s}$ fusion time.

Experimentally, we fabricate five $(1+1) \times 1$ side pumping combiners with different $D_{\text {waist }}$ of the tapered pump fiber but the same tapering length and same heating torch parameter setting. By splicing two of these combiner samples, a two-stage-cascaded side pumping combiner is developed. Table 1 shows different $D_{\text {waist }}$ and pump coupling efficiency of the combiner samples. For the aim of experimentally investigating the influence of $D_{\text {waist }}$ for the first stage on the performance of the component, Samples 1-4 are used as Stage 1 while Sample 5 is for Stage 2. Figure 5(a) shows the diameter of pump fiber tapers for Samples 14 at different longitudinal positions around the taper waist, which is scanned by a CCD. The diameters at the taper waist are different, which are $23 \mu \mathrm{m}, 25 \mu \mathrm{m}, 27 \mu \mathrm{m}$ and $30 \mu \mathrm{m}$, respectively. Slight fluctuations of the diameter, as shown in Figure 5(a), may due to the vibration of motor stages when it is moving during scan testing. Figure 5(b) shows the diameter of the pump fiber taper for Sample 5 at different longitudinal positions scanned by CCD. Here the taper waist is around $25 \mu \mathrm{m}$. The lengths of the transition region and

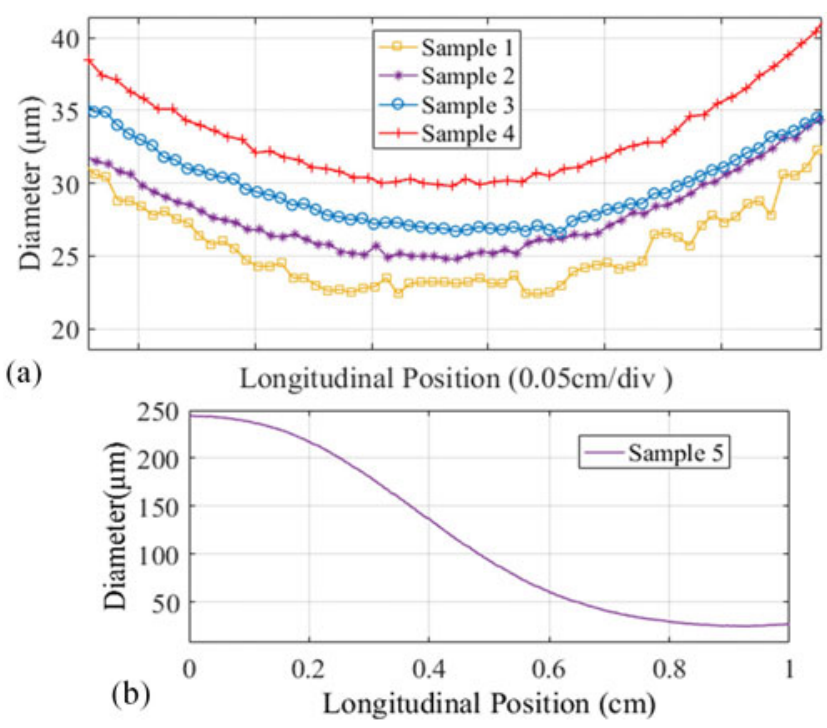

Figure 5. Diameter scanning of pump fiber tapers at different longitudinal positions for (a) Samples 1-4 (around the taper waist) and (b) Sample 5.

Table 1. $D_{\text {waist }}$ and pump coupling efficiency of the combiner samples.

\begin{tabular}{lccc}
\hline Sample & $D_{\text {waist }}(\mu \mathrm{m})$ & Pump coupling efficiency (\%) & Stage \\
\hline 1 & 23 & 97.1 & 1 \\
2 & 25 & 98.1 & 1 \\
3 & 27 & 97.6 & 1 \\
4 & 30 & 91.6 & 1 \\
5 & 25 & 98.0 & 2 \\
\hline
\end{tabular}

the taper waist are $9 \mathrm{~mm}$ and $10 \mathrm{~mm}$, respectively, which are identical to that in simulations.

The experimental setup of pump coupling efficiency testing system for the cascaded side pumping combiner and the microscope images of the combiner is shown in Figure 6. Two laser diodes (LDs) with a maximum output power of $400 \mathrm{~W}$ at the wavelength of $976 \mathrm{~nm}$ are used either for the coupling efficiency test of cascaded structure or every combiner sample (the results are shown in Table 1). The output fiber of the pump diode has a core/clad diameter of $220 / 242 \mu \mathrm{m}$ with an NA of 0.22 . They are spliced to the input pump ports of Stage 1 and Stage 2. The combiner at every stage is fixed on a silica base plate by low-index ultraviolet glue. The silica base plate is set in a component housing which is fixed on a heat sink device (maintaining a constant temperature of $20^{\circ} \mathrm{C}$ by water cooling). The working temperature of the fiber coating of every stage can be monitored by thermal imager.

\subsection{Experimental results}

Figure 7(a) shows the output power versus input pump power for Samples 1-4 as Stage 1 under the condition with only LD1 pumping and the slope efficiency of every 


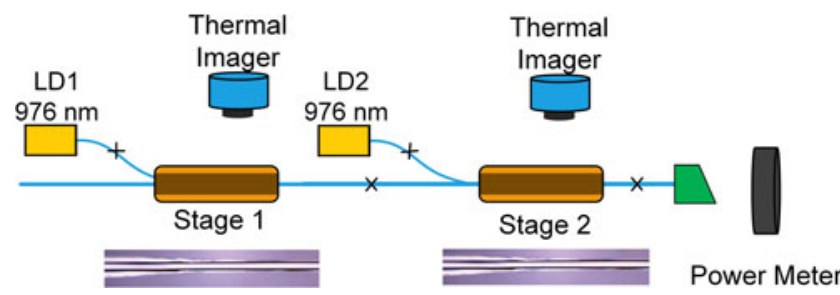

Figure 6. The experimental setup of pump coupling efficiency testing system and the microscope image of the combiner (with LD pumping).

linear fit corresponds to the coupling efficiency (or $\eta_{2}$ in the simulation). A coupling efficiency of $86.5 \%$ is obtained for Sample 3 as Stage 1, which is much higher than that for Samples 1 and 2 as Stage 1. This can be explained by the fact that a larger $D_{\text {waist }}$ of Stage 1 can lead to a higher $\eta_{2}$ as analyzed in Section 2.2. However, when Sample 4 has $D_{\text {waist }}$ of $30 \mu \mathrm{m}$ as Stage 1 , the value of $\eta_{2}$ is only $68.7 \%$, which is due to lower coupling efficiency at Stage 1 before Stage 2 involved (as shown in Table 1), compared with other samples. Figure 7(b) indicates the pump coupling efficiency when LD1 and LD2 pump together, which corresponds to the total coupling efficiency of the two-cascaded scheme. It turns out that the cascaded structure with Sample 3 as Stage 1 has the highest coupling efficiency of $92.3 \%$ than that of other three cases whose total coupling efficiency is not higher than $90 \%$. However, the total coupling efficiencies of Sample 3 and Sample 5 are expected to be $97.8 \%$ ((97.6\% + $98.0 \%) / 2$ ) according to their own coupling efficiency, under the condition without the consideration of the extra loss mechanism of a cascaded structure as analyzed in Section 2. It proves that the loss mechanism of a cascaded scheme will bring challenges for further scaling the power handling capability of a side pumping combiner.

Figure 8 illustrates the highest temperature along the fiber coating of Stage 2 versus different input pump power in the cases of Samples 1-3 as Stage 1. The six data points in Figure 8(a) correspond to the current of LD1 from 1 to $6 \mathrm{~A}$, while those in Figure 8(b) correspond to the current of LD1 and LD2 from 1 to $6 \mathrm{~A}$ simultaneously. As shown in Figure 8(a), with the increase of pump light power from LD1, the highest local temperature on Stage 2 rises with a coefficient of $1.6^{\circ} \mathrm{C} / 10 \mathrm{~W}, 1.5^{\circ} \mathrm{C} / 10 \mathrm{~W}$ and $1.4{ }^{\circ} \mathrm{C} / 10 \mathrm{~W}$ for Samples 1-3 as Stage 1, respectively. This indicates that, in a two-stage-cascaded combiner, samples with different $D_{\text {waist }}$ as Stage 1 show different thermal load on the fiber coating of Stage 2. A smaller $D_{\text {waist }}$ of Stage 1 leads to worse coating thermal load, which agrees well with the numerical results shown in Figure 2(c). As shown in Figure 8(b), compared with the case of only LD1 injected, when LD1 and LD2 inject simultaneously, the coating temperature of Stage 2 is a little higher under the same LD current due to the own temperature rise of Stage 2 with only LD2 injecting (shown
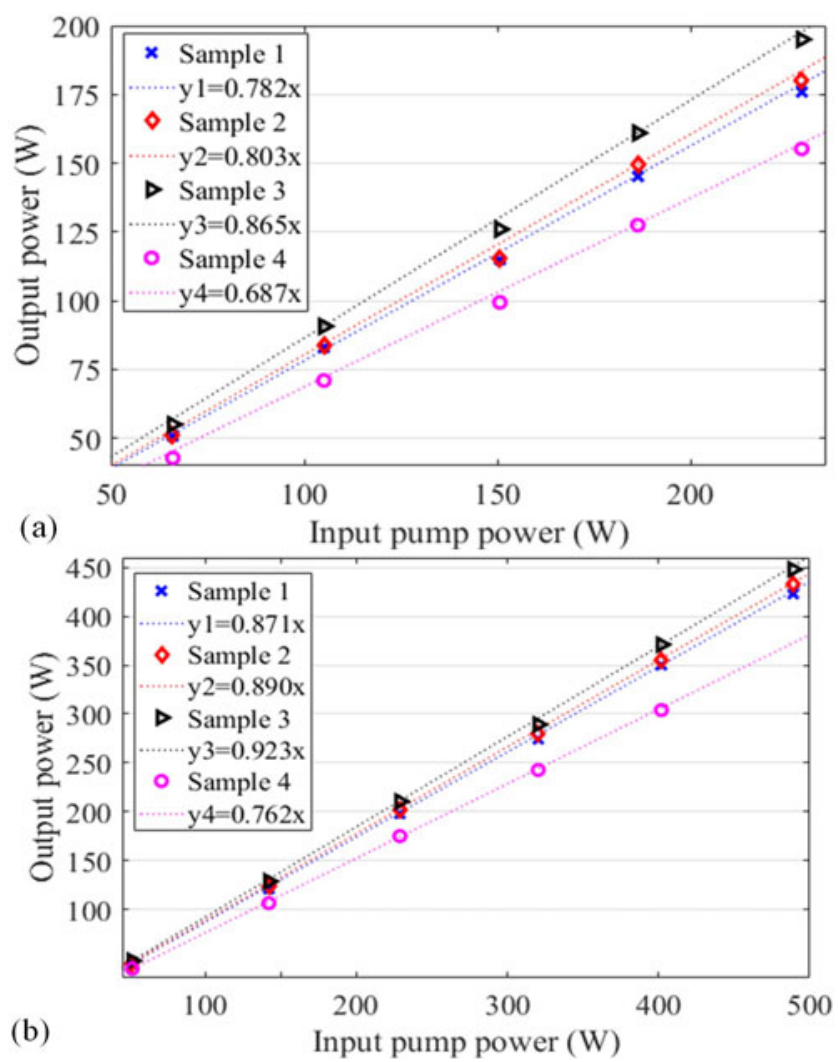

Figure 7. The results of pump coupling efficiency testing for Samples 1-4 as Stage 1. (a) Only with LD1 pumping; (b) with LD1 and LD2 pumping together.

in blue markers of Figure 8(b)). However, compared with its own temperature rise coefficient of only $0.4^{\circ} \mathrm{C} / 10 \mathrm{~W}$, the cascaded structure brings extremely serious thermal load on fiber coating of Stage 2, which will limit the power handling capability of the component.

Figures 9(a) and 9(b) show the thermal images of two stages in the case of Sample 3 and Sample 1 as Stage 1 when $186.3 \mathrm{~W}$ pump light of LD1 and $215.5 \mathrm{~W}$ pump light of LD2 are injected. In order to test the temperature distribution along the fiber coating, line markers are drawn in the analysis software of the thermal imager, which are shown as the white lines in Figure 9. As shown in Figure 9(a), the highest local temperature of the first stage is only $26.1^{\circ} \mathrm{C}$ whereas it is as high as $51^{\circ} \mathrm{C}$ in Stage 2. It can also be observed that the heat load decreases and spreads out gradually along the fiber coating that is packaged in the component housing. This is because the fiber coating is in contact with the silica base plate and a portion of coating loss can be spread by heat sink device. As for the following fiber coating that is not packaged in the combiner housing, the average temperature is tested to be $28.7^{\circ} \mathrm{C}$. Under higher power level, the heat load of this part cannot be ignored because it is naked in the air with no extra cooling management. Figure 9(b) shows the thermal image of the case for Sample 1 as Stage 1. 

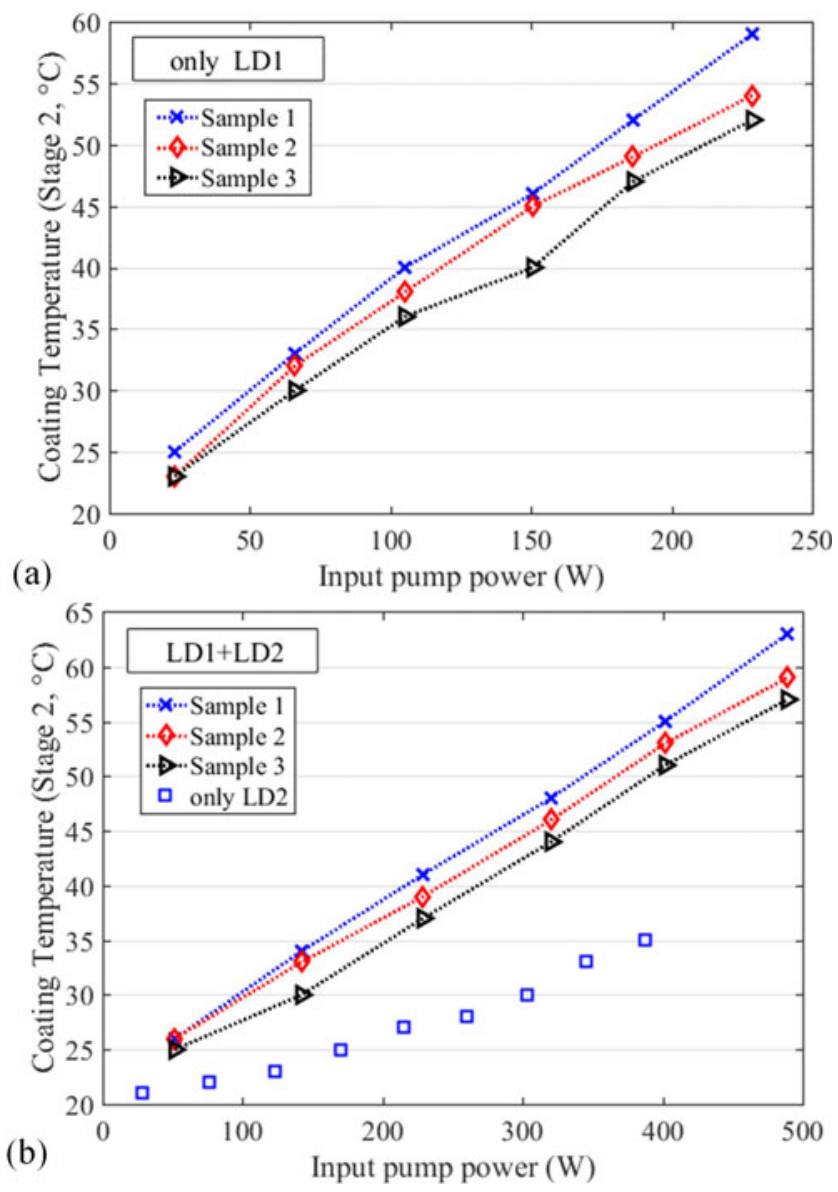

Figure 8. The highest temperature along the fiber coating of Stage 2 for Samples 1-3 as Stage 1: (a) only LD1 injecting pump power or (b) LD1 together with LD2 injecting pump power (the blue square stands for the case that only LD2 injected).

Compared with the result in Figure 9(a), either the heat load on Stage 2 or on Stage 1 is worse than that in Figure 9(a) because of the smaller $D_{\text {waist }}$ of Sample 1 as Stage 1 .

It should be noted that the pump coupling efficiency and heat load on the fiber coating were proved to be significantly influenced by the pump light input NA numerically and experimentally ${ }^{[7,9]}$. In a cascaded structure, especially in the latter stage, this impact will be enhanced because of the further increase of NA along the coupling region. Therefore, for a cascaded side pumping combiner, a pump light source with lower NA or high brightness is advisable in order to achieve a high pump coupling efficiency and low heat load on the fiber coating. Based on the tandempumping scheme, we have recently investigated a $(2+1) \times$ 1 side pumping combiner numerically and experimentally for high power fiber lasers ${ }^{[17]}$. The results imply that the side pumping combiner can have a higher power loading capability with high coupling efficiency when applied in a tandem-pumping scheme due to the high brightness of the fiber laser as the pump light source. In this work, we also use a $1018 \mathrm{~nm}$ fiber laser to act as the pump light source

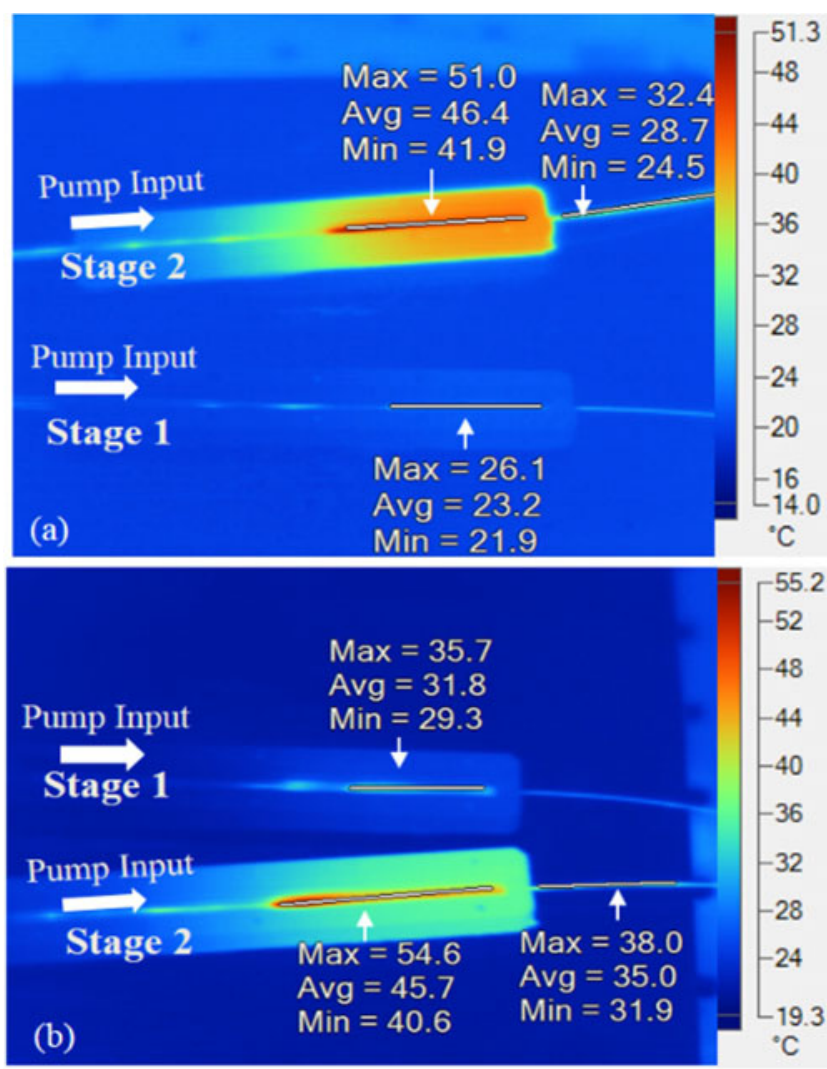

Figure 9. The thermal images of the cascaded combiner when injecting 186.3 W pump light of LD1 and 215.5 W pump light of LD2. (a) Sample 3 as Stage 1; (b) Sample 1 as Stage 1.

of the cascaded combiner and the experimental setup is shown in Figure 10(a). Seven ytterbium-doped fiber lasers with the center wavelength of $1018 \mathrm{~nm}$ are coupled to a multi-mode fiber $(200 / 220 \mu \mathrm{m}, \mathrm{NA}=0.22)$ by a $7 \times 1$ combiner. Sample 3 and Sample 5 act as Stage 1 and Stage 2 in the cascaded scheme. Figure 10(b) shows the thermal image of the two stages when $1088 \mathrm{~W} 1018 \mathrm{~nm}$ laser light is injected (545 W for Stage 1 and $543 \mathrm{~W}$ for Stage 2). The corresponding output power is $1048 \mathrm{~W}$, obtaining the total coupling efficiency of $96.4 \%$. The highest temperature along the coating of Stage 1 is only $23.2^{\circ} \mathrm{C}$, which means the combiner shows an excellent power handling capability in a one-stage structure. However, the highest temperature along the coating of Stage 2 is $44.2^{\circ} \mathrm{C}$, even though this performance is much better compared with $\mathrm{LD}$ as pump source shown in Figure 9(a). The results indicate that, despite of the application of fiber lasers as the pump source, the loss mechanism induced by a cascaded structure cannot be ignored. We believe that the coupling efficiency and the thermal load of the fiber coating could be optimized if the core diameter of the multi-mode delivery fiber for the signal combiner, which combines several $1018 \mathrm{~nm}$ Yb-doped fiber lasers together, is smaller. 


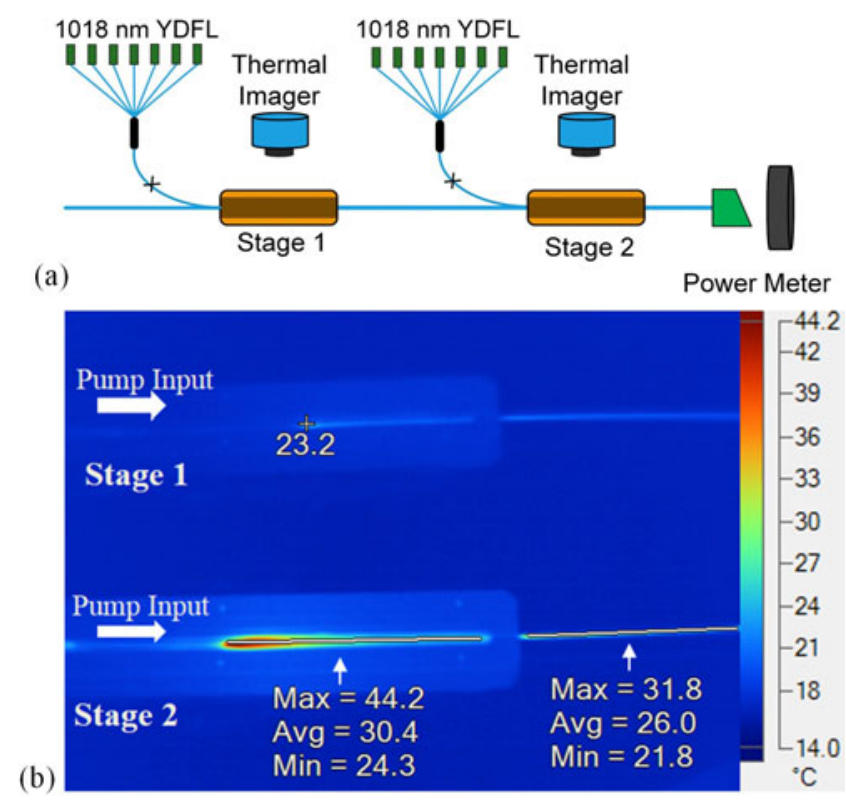

Figure 10. (a) Experimental setup of the component test with $1018 \mathrm{~nm}$ fiber laser pumping. (b) The thermal image of the cascaded combiner when injecting total $1018 \mathrm{~nm}$ laser light of $1088 \mathrm{~W}$ (545 W for Stage 1 and $543 \mathrm{~W}$ for Stage 2). Stage 1/Stage 2: Sample 3/Sample 5.

\subsection{Discussion}

The experiments reveal that first, the total coupling efficiency of a two-cascaded side pumping combiner is lower than expected, due to the power loss along the coupling region of the latter stage as well as the enhanced LPC in the latter stage, derived from light power launching in Stage 1. Second, the heat load in Stage 2 is more serious than that in Stage 1 even only with pump light injecting to Stage 1, which is the critical limit of the power handling capability for the cascaded structure. Moreover, one should take more effort to handle the heat load on the signal fiber coating of the latter stage rather than the former one. Third, a larger $D_{\text {waist }}$ of the tapered pump fiber in the former stage is advantageous to reduce the thermal load on the signal fiber coating of the latter stage while only under the condition that the coupling efficiency of the former stage is not decreased due to the variation of $D_{\text {waist }}$. The experiments also prove that with the option of using fiber laser as the pump light source, the future opportunities for further improving the performance of a cascaded side pumping combiner, including the total coupling efficiency and the pump power handling capability, appear very bright. A three-stage-cascaded side pumping combiner is also feasible as long as the tapering structure (the taper length and taper waist diameter) and fusion splicing parameter (heating torch size, heating region size, heating temperature) can be well designed through numerical calculation. Even so, our work indicates that the extra pump power loss due to the cascaded scheme cannot be ignored and will cause the decrease of total pump coupling efficiency. Thus, the number of stage is limited by the expected total pump coupling efficiency from users. To further explore the advantage of unlimited pump points for side pumping combiners, a multi-point distributed pumping scheme is also of great potential where the pump fibers are fusion-spliced on the surface of the active fiber directly. In this scheme, the pump power coupled from the former stage of the combiner will be absorbed by the raredoped signal fiber core and only a portion of the unabsorbed pump light propagates to the coupling region of the next stage, which means the extra pump power loss due to the cascaded structure will significantly decrease. Meanwhile, the inhomogeneous gain distribution and thermal effects occurring at the end of the fiber can also be solved by this scheme.

\section{Conclusion}

In conclusion, we have investigated the loss mechanism of a two-stage-cascaded side pumping combiner numerically and experimentally. The theoretical analysis indicates that the coupling efficiency is lower than the expected due to the extra power loss when the pump light from the first stage propagates to the next stage, and the LPC on the coating of the second stage is more serious than that in the first stage. Experimentally we fabricate combiners consisting of one pump fiber $(220 \mu \mathrm{m} / 242 \mu \mathrm{m}, \mathrm{NA}=0.22)$ and a signal fiber $(20 \mu \mathrm{m} / 400 \mu \mathrm{m}, \mathrm{NA}=0.46)$ and splice two of them to construct a cascaded scheme. The influences of $D_{\text {waist }}$ of the tapered pump fiber in the first stage on the thermal load and coupling efficiency of the components are monitored and analyzed. The experiments show good agreement with the BPM simulations. Furthermore, $1018 \mathrm{~nm} \mathrm{Yb-doped}$ fiber lasers are also adopted to be the pump sources of the combiner. The maximum power handling of $1088 \mathrm{~W}$ with a pump coupling efficiency of $96.4 \%$ is achieved for a two-cascaded combiner, which shows its advantage in further improving the power handling capacity of a cascaded structure.

\section{Acknowledgements}

This work was supported by the National Natural Science Foundation of China (No. 61370045) and the National Key R\&D Program of China (No. 2017YFF0104600).

\section{References}

1. D. J. Digiovanni and A. J. Stentz, Tapered fiber bundles for coupling light into and out of cladding-pumped fiber devices, U.S. Patent 5864644 (1999).

2. T. Weber, W. Lüthy, and H. P. Weber, Appl. Phys. B 63, 131 (1996).

3. L. Goldberg, B. Cole, and E. Snitzer, Electron. Lett. 33, 2127 (1997).

4. J. P. Koplow, S. W. Moore, and D. A. V. Kliner, IEEE J. Quantum Electron. 39, 529 (2003). 
5. S. L. Lin, Y. W. Lee, K. Y. Hsu, and C. W. Huang, in Lasers and Electro-Optics Pacific Rim (2013), paper TuA1-5.

6. C. W. Huang, D. W. Huang, C. L. Chang, D. Y. Jheng, K. Y. Hsu, C. H. Kuan, and S. L. Huang, in CLEO: 2011 - Laser Science to Photonic Applications (2011), paper JWA41.

7. T. Theeg, H. Sayinc, J. Neumann, L. Overmeyer, and D. Kracht, Opt. Express. 20, 28125 (2012).

8. X. Chen, Q. R. Xiao, G. Y. Jin, P. Yan, and M. L. Gong, Chin. Phys. B 24, 064208 (2015).

9. Q. R. Xiao, P. Yan, H. C. Ren, X. Chen, and M. L. Gong, J. Lightwave Technol. 31, 2715 (2013).

10. C. Lei, Z. Chen, J. Leng, Y. Gu, and J. Hou, J. Lightwave Technol. 35, 1922 (2017).
11. Q. Xiao, X. Chen, H. Ren, P. Yan, and M. Gong, Opt. Lett. 38, 1170 (2013).

12. Q. Tan, T. Ge, X. Zhang, and Z. Wang, Laser Phys. 26, 25102 (2016).

13. J. Zheng, W. Zhao, B. Zhao, Z. Li, C. Chang, G. Li, Q. Gao, P. Ju, W. Gao, S. She, P. Wu, C. Hou, and W. Li, Opt. Eng. 57, 36105 (2018).

14. S. Zou, H. Chen, H. Yu, J. Sun, P. Zhao, and X. Lin, Appl. Phys. B 123, 288 (2017).

15. Q. Xiao, P. Yan, H. Ren, X. Chen, and M. Gong, Appl. Opt. 52, 409 (2013).

16. M. D. Feit and J. A. Fleck, Appl. Opt. 17, 3990 (1978).

17. Y. Gu, C. Lei, J. Liu, R. Li, L. Liu, and H. Xiao, Opt. Eng. 56, 11619 (2017). 